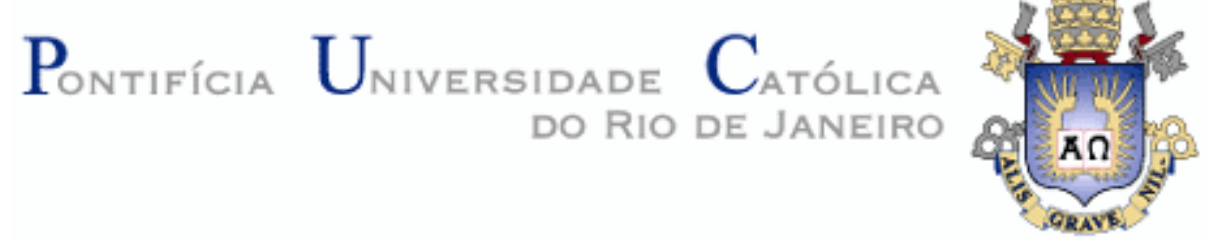

Lívia Fonseca de Medeiros Silva

\title{
O IMPACTO DO WAREHOUSE MANAGEMENT SYSTEM (WMS) NOS INDICADORES DE DESEMPENHO LOGÍSTICO: APLICAÇÃO EM CENTRO DE DISTRIBUIÇÃO DE MEDICAMENTOS
}

\section{Dissertação de Mestrado}

Dissertação apresentada como requisito parcial para obtenção do grau de Mestre pelo Programa de Pós-Graduação em Engenharia de Produção do Departamento de Engenharia Industrial da PUCRio.

Orientador: Prof. Nélio Domingues Pizzolato 


\section{O IMPACTO DO WAREHOUSE MANAGEMENT SYSTEM (WMS) NOS INDICADORES DE DESEMPENHO LOGÍSTICO: APLICAÇÃO EM CENTRO DE DISTRIBUIÇÃO DE MEDICAMENTOS}

Dissertação apresentada como requisito parcial para obtenção do grau de Mestre pelo Programa de Pós-Graduação em Engenharia de Produção PUC-Rio. Aprovada pela Comissão Examinadora abaixo assinada.

Prof. Nélio Domingues Pizzolato

Orientador

Departamento de Engenharia Industrial - PUC-Rio

Prof. José Eugêncio Leal

Departamento de Engenharia Industrial - PUC-Rio

Profa. Valeria Campos Gomes de Souza Micucci

Centro de Análise e Sistemas Navais - CASNAV

Prof. José Eugêncio Leal

Coordenador Setorial do Centro Técnico Científico - PUC-Rio

Rio de Janeiro, 5 de setembro de 2012 
Todos os direitos reservados. É proibida a reprodução total ou parcial do trabalho sem a autorização da universidade, da autora e do orientador.

\section{Lívia Fonseca de Medeiros Silva}

Graduou-se em Engenharia de Produção na UENF (Universidade Estadual do Norte Fluminense) em 2008 e pós-graduou-se em Gestão de Negócios e Inteligência Competitiva pela UNI-RIO em 2011. Atualmente trabalha em consultoria na área de sistemas de gerenciamento logístico.

Ficha Catalográfica

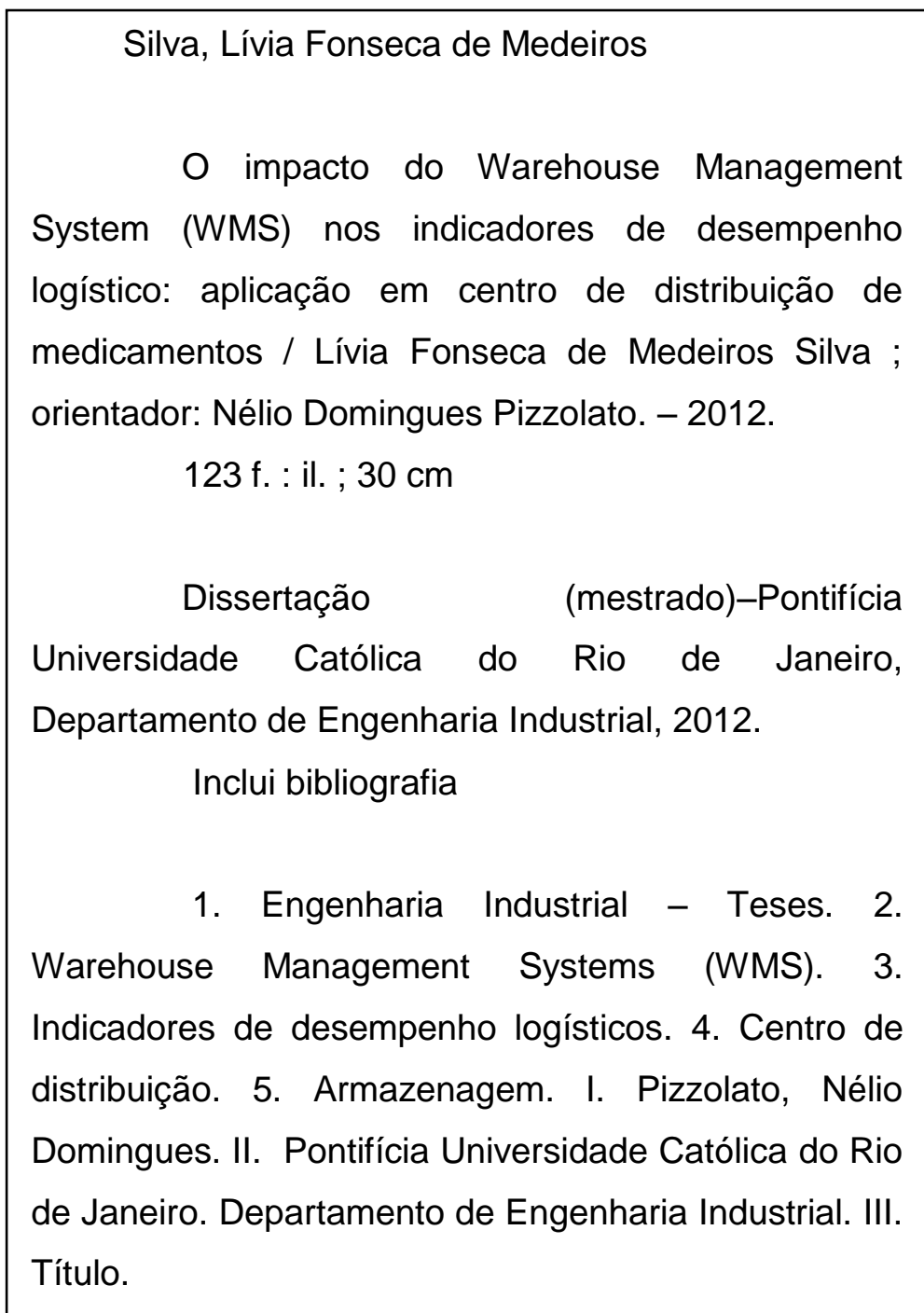

CDD: 658.5 
Aos meus pais Elizabeth e Emílio pelo apoio e carinho. E ao meu irmão Rafael por estar ao meu lado. 


\section{Agradecimentos}

Aos meus pais que sempre me incentivaram ao longo da minha vida, por todo amor e carinho. Ao meu irmão pelo apoio e atenção.

Ao meu orientador Prof. Nélio Domingues Pizzolato pela confiança depositada em mim e por acreditar na minha capacidade para a realização deste trabalho.

A CAPES e à PUC-RIO, pelos auxílios concedidos, sem os quais este trabalho não poderia ter sido realizado.

Aos professores José Eugênio Leal e Valéria Micucci que participaram da Comissão Examinadora.

Aos amigos que fiz durante o mestrado, em especial Augusto, Bárbara, Juliana, Nayara e David que de alguma forma colaboraram para este projeto.

A equipe de projetos de WMS da empresa em estudo, Cristiano, Fernando, Alan, Fabrízio e Vinicius pelo auxilio e compreensão da importância desta dissertação.

A todos os professores e funcionários do Departamento de Engenharia Industrial.

A todos que contribuíram para este trabalho. 


\section{Resumo}

Silva, Lívia Fonseca de Medeiros; Pizzolato, Nélio Domingues (orientador). O Impacto do Warehouse Management System (WMS) nos Indicadores de Desempenho Logístico: Aplicação em Centro de Distribuição de Medicamentos. Rio de Janeiro, 2012. 123p. Dissertação de Mestrado - Departamento de Engenharia Industrial, Pontifícia Universidade Católica do Rio de Janeiro.

A competitividade econômica tem levado as empresas a buscarem novas soluções através do uso de Tecnologia de Informação (TI) para alcançar vantagens competitivas frente a seus concorrentes. O uso da TI permite às empresas terem maior controle na gestão de seus processos e, com isso, obter ganhos de produtividade, redução de custos operacionais e satisfação dos clientes. O presente estudo tem como objetivo apresentar e analisar o processo de implementação de um Sistema de Gerenciamento de Armazéns - WMS em atividades logísticas em um centro de distribuição (CD). Será feito um estudo de caso, que consiste em uma avaliação pós-implementação do sistema em um centro de distribuição de medicamentos através de aplicação de um Sistema de Medição de Desempenho Logístico, a fim de identificar as melhorias e ganhos nas atividades de um armazém. Dentro desse contexto, são identificados também os riscos e as resistências na aplicação do WMS. A metodologia utilizada para esta dissertação é de natureza qualitativa, que consiste em revisão bibliográfica, e de caráter exploratório, mediante pesquisas de campo, visitas na empresa do estudo e entrevistas. O resultado deste estudo é uma análise da melhoria de desempenho após a aplicação do WMS, que constata vantagens da TI para a logística de distribuição como maior nível de acuracidade e redução de tempo.

\section{Palavras-chave}

Warehouse Management Systems (WMS); Indicadores de Desempenho Logísticos; Centro de Distribuição; Armazenagem. 


\section{Abstract}

Silva, Lívia Fonseca de Medeiros; Pizzolato, Nélio Domingues (Advisor). The Impact of Warehouse Management System (WMS) on the Logistics Performance Indicators: Implementation in Pharmaceutical Distribution Center. Rio de Janeiro, 2012. 123p. MSc. Dissertation Departamento de Engenharia Industrial, Pontifícia Universidade Católica do Rio de Janeiro.

The economic competitiveness has led companies to seek new solutions through the use of Information Technology (IT) to achieve competitive advantages over their competitors. The use of IT enables companies to have greater control in managing their processes and thereby achieve productivity gains, reduce operating costs and improve customer satisfaction. The present study aims to present and analyze the process of implementing a Warehouse Management System - WMS on logistics activities in a distribution center (DC). There will be a case study, consisting of a post-implementation review of the system in a distribution center for drugs through implementation of a Performance Measurement System Logistics in order to identify improvements and gains in the activities of a warehouse. Within this context, are also identified risks and resistance in the implementation of WMS. The methodology used for this thesis is qualitative in nature, consisting of a literature review, and exploratory character, through field research, visits to the company in the are study and interview. The result of this study is an analysis of performance improvement after applying WMS, noting advantages of IT for logistics distribution such as a higher level of accuracy and time reduction.

\section{Keywords}

Warehouse Management System (WMS); Logistics Performance Indicators; Distribution Center; Warehousing. 


\section{Sumário}

LISTA DE FIGURAS 10

LISTA DE TABELAS 11

LISTA DE ABREVIAÇÕES E SIGLAS 12

1 INTRODUÇÃO 13

1.1 Objetivos 14

1.2 Motivação 14

$\begin{array}{ll}1.3 \text { Metodologia de Pesquisa } & 15\end{array}$

1.4 Estrutura da Dissertação 15

2 LOGÍSTICA E ARMAZENAGEM 16

2.1. Definição de Logística 16

2.2. Logística Integrada 17

2.3. Armazenagem 20

2.3.1. Manuseio de materiais 23

3.CENTRO DE DISTRIBUIÇÃO 26

3.1. Conceito 26

3.2. Centro de Distribuição 28

3.3. Cross docking 29

3.4. Merge in Transit 30

3.5. Transit Point 32

3.6. Funções Básicas de um CD 32

3.6.1. Recebimento 33

3.6.2. Movimentação 34

3.6.3. Estocagem 34

3.6.4. Picking/ Separação 35

3.6.5. Expedição 36

4 WAREHOUSE MANAGEMENT SYSTEMS 37

4.1 Uso da tecnologia da Informação na Cadeia de Suprimentos 37

4.1.1 Investimentos em TI no Brasil 39 
4.2 Enterprise Resource Planning - ERP 40

4.3 Warehouse Management Systems 41

4.3.1 Implantação WMS 47

5 SISTEMA DE MEDIÇÃO DE DESEMPENHO LOGÍSTICO 49

5.1 Conceitos de Medição de Desempenho 49

5.1.1 Indicadores de desempenho 50

5.2 Sistema de Medição de Desempenho (SMD) 51

5.3 Evolução do SMD: Tradicionais aos Novos Modelos 53

5.4 Modelos de Sistema de Medição de Desempenho 55

5.5 Processo de Desenvolvimento e Implementação de SMD 63

5.6 Medição de Desempenho na Logística 66

5.7 Modelos de Medição de Desempenho Logístico 67

5.8 Definição do SMD para pós-implantação do WMS 73

6 ESTUDO DE CASO 76

6.1 Setor Farmacêutico 76

6.2 Estudo de Caso Empresa A 80

6.2.1 Escolha do Warehouse Management System - WMS 83

6.2.2 Definição do Fluxo de Operações 85

6.2.3 Fase da Implementação 88

6.2.4 Aplicação dos Indicadores e Resultados 96

7 CONCLUSÃO 109

7.1 Sugestões para Trabalhos Futuros 111

REFERÊNCIAS BIBLIOGRÁFICAS 112

ANEXO I 119

ANEXO II $\quad 120$

ANEXO III 121

ANEXO IV 122

ANEXO V 123 


\section{Lista de Figuras}

Figura 2.1 Integração Logística 20

Figura 3.1 Atividades de Ciclo Básico da Distribuição Física 28

Figura 3.2 Fluxo do Merge in Transit 32

Figura 3.3 Fluxo dentro de um CD 34

Figura 4.1 Sistema de Informações Logística 39

Figura 5.1 Processo de Gestão de Desempenho e a Posição de Medição de Desempenho 53

Figura 5.2 Modelo SMART $\quad 57$

Figura 5.3 O modelo Balanced Scorecard $\quad 59$

Figura 5.4 Relacionamento entre Medidas de Desempenho 60

Figura 5.5 Modelo Quantum 63

Figura 5.6 Processo de Desenvolvimento de Sistemas de Medição de Desempenho 65

Figura 5.7 Fases do Processo de Medição de Desempenho 66

Figura 5.8 Modelo World Class Logistic 69

Figura 6.1 Processo Físico da Cadeia de Suprimentos da Indústria Farmacêutica $\quad 78$

Figura 6.2 Layout do CD da empresa A 83

Figura 6.3 Fases da Implementação do Projeto 90

Figura 6.4 Estrutura do Endereço de Pulmão 91

Figura 6.5 Estrutura do Endereço de Flowrack 92

Figura 6.6 Taxa de Atendimento - Abril $2012 \quad 99$

Figura 6.7 Taxa de Atendimento (\%) - Maio $2012 \quad 99$

Figura 6.8 Taxa de Atendimento (\%) - Junho 2012

Figura 6.9 Percentual de Entregas no Prazo Abril (\%) 101

Figura 6.10 Percentual de Entregas no Prazo Maio (\%) 102

Figura 6.11 Percentual de Entregas no Prazo Junho (\%) 102

Figura 6.12 Tempo Médio de Embarque (horas) 103

$\begin{array}{ll}\text { Figura 6.13 Acuracidade do Lote } & 105\end{array}$

Figura 6.14 Unidades de Caixa Fechada Separadas/Hora 106

Figura 6.15 Unidades de Caixa Fechada Separadas/Hora 107 


\section{Lista de Tabelas}

Tabela 2.1 Armazém Tradicional X Moderno 22

Tabela 4.1 Características e Funcionalidades WMS 43

Tabela 5.1 Matriz Quantum de Medição de Desempenho 62

$\begin{array}{ll}\text { Tabela 5.2 Definição de Indicadores } & 67\end{array}$

Tabela 5.3 Dúvidas Gerência Logística 72

Tabela 5.4 Indicadores para avalição do WMS 76

Tabela 6.1 Problemas Identificados antes da Implementação 84

Tabela 6.2 Check List Go Live 96

Tabela 6.3 Indicadores Aplicados 98

Tabela 6.4 Comparativo Antes e Depois 107 


\section{Lista de Abreviaturas e Siglas}

ABC - Activity Based Costing

ANVISA - Agência Nacional de Vigilância Sanitária

$\mathrm{BI}$ - Business Intelligence

BSC - Balance Scorecard

CD - Centro de Distribuição

EDI - Electronic Data Interchange

ERP - Enterprise Resource Planning

FIFO- First in First Out

FPNQ - Fundação Prêmio Nacional de Qualidade

GPS - Global Positioning System

LTL - Less Than Truck

MRP I - Material Requirement Planning

MRP II - Manufacturing Resource Planning

PROVAR - Programa de Administração de Varejo

RFDC - Radio Frequency Data Communications

RFID - Radio Frequency Identification

$\mathrm{ROI}$ - Return on Investment

SMART - Strategic Measurement, Analysis, and Reporting Technique

SMD - Sistema de Medição De Desempenho

TI - Tecnologia de Informação

TMS - Transport Management System

WCL - World Class Logistic

WCS - Warehouse Control System 